\title{
Difficult diagnosis of Infliximab-related miliary tuberculosis
}

\author{
S. Contini1 , G. Raimondi1,2, P. Graziano ${ }^{3}$, C. Saltini1 ${ }^{1}, 2$ M. Bocchino 2
}

ABSTRACT: Difficult diagnosis of Infliximab-related miliary tuberculosis. S. Contini, G. Raimondi, P. Graziano, C. Saltini, M. Bocchino.

The use of Infliximab in the treatment of patients with rheumatoid diseases unresponsive to conventional therapies has been reported to be complicated by opportunistic infections. We report the case of a 56-yr old female rheumatoid arthritis patient complaining of fever and respiratory symptoms 9 months after commencing Inflix- imab, who received no ethiologic diagnosis for the six months before admission. Tuberculosis was suspected upon chest radiographic pictures and empirical treatment for miliary tuberculosis instated in the wake of microbiological confirmation. The case typifies the difficulties of diagnosing miliary tuberculosis in the immunocompromised as well as in the immunocompetent patient.

Monaldi Arch Chest Dis 2004; 61: 2, 128-130.

Keywords: TNF- $\alpha$, Infliximab, miliary tuberculosis.

${ }^{1}$ Cattedra di Malattie Respiratorie e Scuola di Specializzazione in Malattie Respiratorie, Università Tor Vergata, Roma, Italy; ${ }^{2}$ Divisione Clinicizzata di Malattie Respiratorie INMI L. Spallanzani-IRCCS, Roma, Italy; 3 U.O. di Anatomia ed Istologia Patologica, Ospedale C. Forlanini, A.O. San Camillo-Forlanini, Roma, Italy.

Correspondence: Dr Marialuisa Bocchino; Divisione Clinicizzata di Malattie Respiratorie INMI L. Spallanzani-IRCCS, Via Portuense 292,00149, Roma, Italy; e-mail: mlbocchino@tiscali.it

\section{Introduction}

Anti-tumour necrosis factor (TNF)- $\alpha$ or antiTNF- $\alpha$ receptor was introduced in 1998 as treatment for rheumatoid arthritis (RA) unresponsive to conventional immunosuppressive drugs [1, 2]. Since TNF- $\alpha$, a pleiotropic cytokine produced by macrophages and lymphocytes exerting powerful pro-inflammatory effects, plays a critical role in response to infections [3], anti-TNF- $\alpha$ treatment has been complicated by serious opportunistic infections [4]. Among the adverse effects reported are at least eight cases of candidiasis, seventeen of listeriosis, ten of $P$. carinii pneumonia, eleven of histoplasmosis, seven of aspergillosis, one case of cryptococcosis, two of Salmonella septicemia, one case of severe pneumococcal pneumonia and 70 cases of microbiologically-confirmed tuberculosis (TB) in Infliximab treated patients with rheumatoid arthritis, Crohn's disease and ankylosing spondylitis. In patients with TB, the median interval between the beginning of treatment and the onset of TB was 12 weeks [5].

\section{Case report}

A 56-year-old non-smoking female homemaker, was admitted in December, 2001, with a 6month history of fever of unknown origin. Her medical history was remarkable for pleural TB at the age of 11 years and for a 12-year history of severe RA unresponsive to both steroidal and nonsteroidal anti-inflammatory drugs. She had commenced Infliximab 15 months prior to admission, with a monthly-single dose regimen of $3 \mathrm{mg} / \mathrm{Kg}$. Treatment was terminated after 9 months, due to persistent fever. Upon admission, the patient reported two previous hospitalisations for fever, treated with corticosteroids and broad-spectrum antibiotics, without any apparent benefit. She also reported having had two chest X-rays taken at six and at two months prior to admission, both read as normal.

Upon admission, she complained of moderate dyspnoea on exertion and dry cough. Body temperature was $38.5 \mathrm{C}^{\circ}$. Physical exam of the chest revealed bi-basilar inspiratory crackles. Chest X-ray showed bilateral profusion of small, sub-centimetric nodules (figure 1), appearing as well-defined, 2$4 \mathrm{~mm}$ opacities with upper lobe prevalence on high resolution computed tomography (HRCT) (figure 2 A-D). The tuberculin skin test (PPD, 5 IU) was negative. Routine laboratory tests run on admission showed an increase of the erythrocyte sedimentation rate $(79 \mathrm{~mm} / \mathrm{h})$ with relative neutrophilia (total white cell count $=9.2 \times 103 / \mathrm{mm}^{3}$; neutrophils $=$ $83 \%$ ). The peripheral CD4/CD8 $\mathrm{T}$ cell ratio was 3.2. Microbiological examination of sputum was negative for acid-fast bacilli (AFB), fungi, and other microorganisms, including $P$. carinii. Blood samples were drawn for AFB culture. Fiberopticbronchoscopy (FBS) was not performed due to a significant low oxygen saturation $\left(\mathrm{SaO}_{2}\right)$ levels. The ECG showed diffuse alterations in repolarisation, such as $\mathrm{T}$ wave inversion in leads V1-V3, suggestive of antero-septal ischemia.

Based upon the findings of miliary nodule dissemination on HRCT, the patient was begun em- 


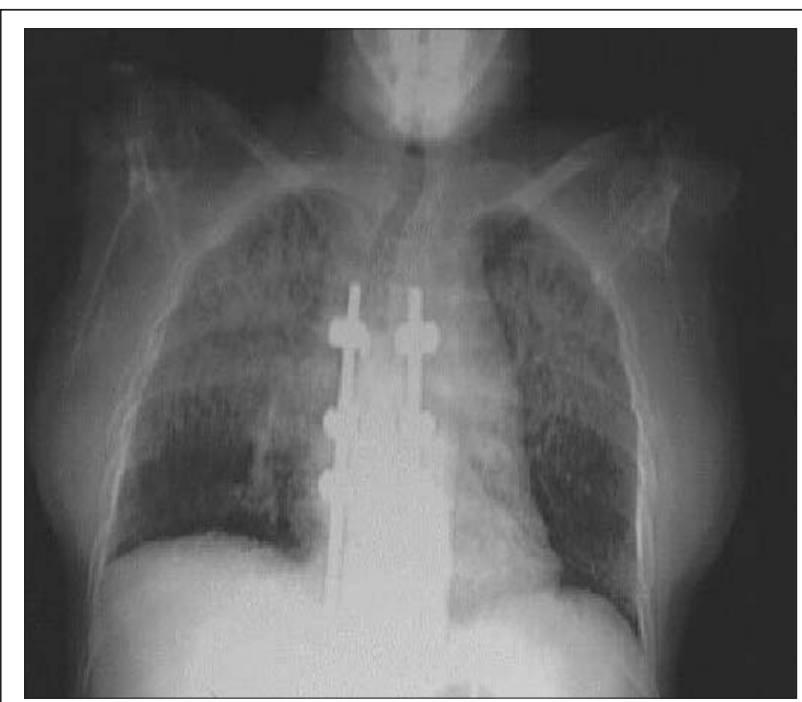

Fig. 1. - Standard chest $\mathrm{x}$-ray at admission showing bilateral, predominanantly upper lobe profusion of smale sub-centimetric nodules.

pirically on standard anti-TB therapy with Isoniazid $(5 \mathrm{mg} / \mathrm{kg})$, Rifampin $(10 \mathrm{mg} / \mathrm{kg})$, Ethambutol (25 $\mathrm{mg} / \mathrm{kg}$ ) and Pyrazinamide $(25 \mathrm{mg} / \mathrm{kg})$. Her general medical condition improved and the fever remitted after 10 days of treatment. Three weeks later, the patient died of acute heart failure.

Upon autopsy, myocardial infarction of the left ventricle was found as the cause of death in the context of severe coronary arteriosclerosis. Histopathological examination of the lungs showed the presence of multiple, both isolated and confluent, millimetric, organized granulomas with prominent caseous necrosis. Stains for acid-fast bacilli were positive (figure 3). A blood sample resulted positive for M. tuberculosis after 5 weeks of culture.
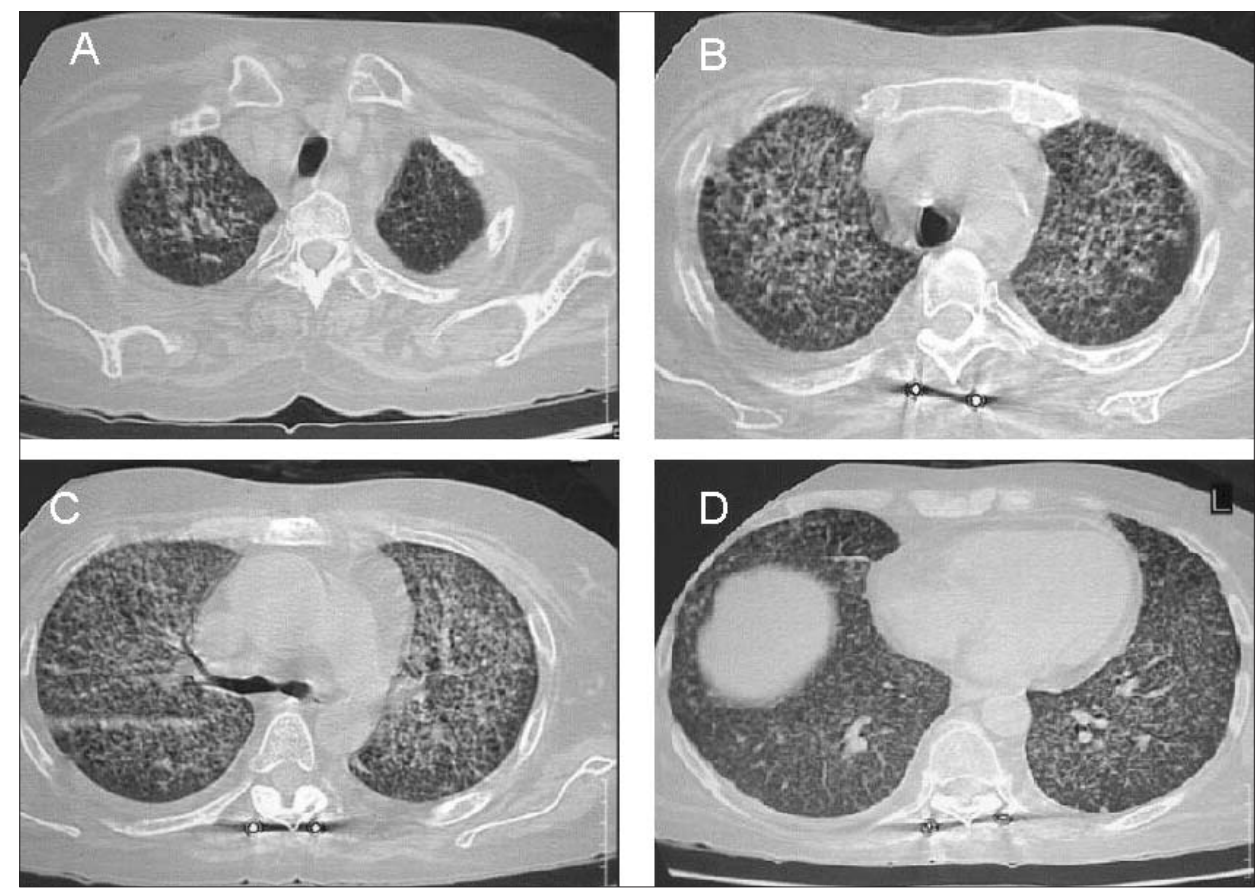

Fig. 2. - Selected images (A-D) of the HRCT of the thorax showing bilateral distribution of well-defined 2-4 $\mathrm{mm}$, micro-nodular interstitial opacities, with upper lobe prevalence.

\section{Discussion}

Here we describe a case of miliary TB complicating anti-TNF- $\alpha$ Infliximab treatment in which a post mortem histological diagnosis was confirmed by blood culture. In the presence of negative sputum exams, anti-TB treatment was started based upon chest X-ray and HRCT images highly indicative of miliary tuberculosis. Infliximab is a humanised anti-TNF- $\alpha$ monoclonal antibody with a half life of 10 days. The biological effects persist for up to 2 months, thus it may be administered in a single dose $(3 \mathrm{mg} / \mathrm{Kg})$ monthly-regimen, or on day 0 , day 14 and day 42 and then every 8 weeks thereafter [6]. In the case herein described, fever developed after nine months of Infliximab treatment and the chest X-ray did not show the typical miliary TB's bilateral micronodular opacities until six months after the appearance of low grade fever. In the previously reported cases, TB developed within 3 months of initiation of the drug [5]. In this respect, although $\mathrm{TB}$, notably both pulmonary and systemic, has been described in association with the use of Infliximab with a much higher frequency than any other opportunistic infection, the late appearance of fever and respiratory symptoms may have misled physicians attending in this case.

In fact, miliary TB is not an unusual outcome of TB reactivation complicating anti-TNF- $\alpha$ treatment, and it has been difficult to diagnose. In the series of Keane et al., extra-pulmonary TB was found in $56 \%$ of cases. Twenty four percent of them had disseminated disease [5] and $47 \%$ required a diagnostic tissue biopsy. In this regard, in the three cases of Infliximab-related TB where a miliary pattern has been clearly described [7-9], BAL and sputum cultures were obtained in only two cases and the diagnosis was made at autopsy in one. In one case, symptoms preceded the appearance of the typical miliary $\mathrm{X}$-ray pattern by 6 weeks [7].

It is not unusual that the diagnosis of miliary TB is either tardy or missed altogether $[10,11]$. In a series report of 38 non-immunocompromised adults with miliary $\mathrm{TB}, \operatorname{six}(16 \%)$ were reported to have presented with negative chest X-rays: the diagnosis was obtained by laparotomy in four cases and at autopsy in two. An eighteen percent mortality rate was reported in this series [12]. Similarly, among the 44 cases of miliary TB reported by Ibanez 


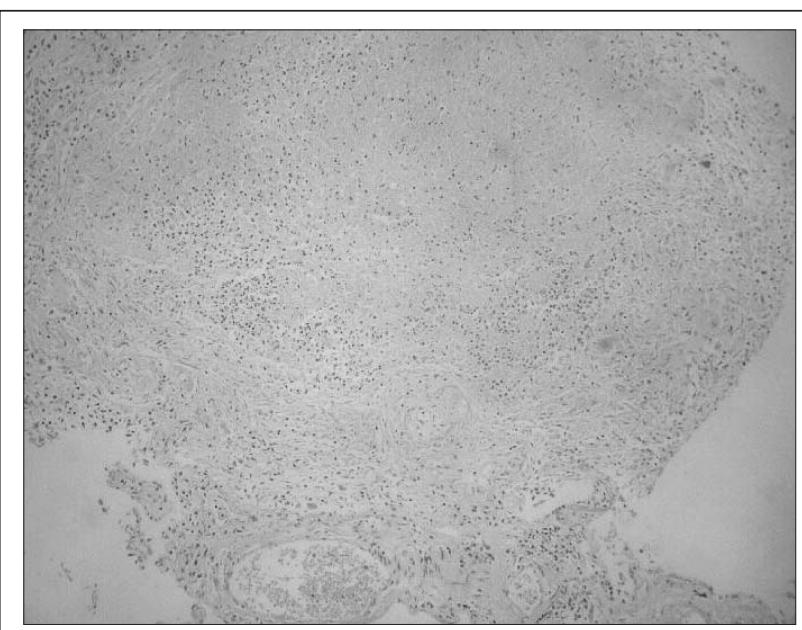

Fig. 3. - On microscopic examination of the lung parenchyma, multiple, both isolated and confluent, epitheliod necrotizing granulomas with scattered giant cells were observed (H\&E 4X). In situ acid-fast stain for mycobacteria (not shown) was positive.

Martinez et al., the diagnosis was obtained at autopsy in 32 [13].

In miliary $\mathrm{TB}$, the intensity of the elimination of TB bacilli in spontaneously produced sputum samples is reportedly very low. Blood cultures have been found the most sensitive tool for the diagnosis of TB in immunocompromised subjects [14], where pulmonary TB may present in the absence of radiological findings and miliary $\mathrm{TB}$ is frequently associated with higher degrees of immunodeficiency $[15,16]$. Blood cultures, together with CT scans, may provide highly indicative results and help avoiding more aggressive interventions.

\section{References}

1. Schema AA, Megeff C. New drugs for the treatment of rheumatoid arthritis. Am J Health Syst Pharm 2000; 57: 225-234.

2. Criscione LG, St Clair EW. Tumor necrosis factor-alpha antagonists for the treatment of rheumatic diseases. Curr Opin Rheumatol 2002; 14: 204-211.

3. Knight JC, Kwiatkowski D. Inherited variability of tu- mor necrosis factor production and susceptibility to infectious disease. Proc Assoc Am Physicians 1999; 111: 290-298.

4. Bresnihan B, Cunnane G. Infection complications associated with the use of biologic agents. Rheum Dis Clin North Am 2003; 29: 185-202.

5. Keane J, Gershon S, Wise RP, et al. Tuberculosis associated with infliximab, a tumor necrosis factor $\alpha$-neutralizing agent. $N$ Engl J Med 2001; 345: 1098-1104.

6. Remicade for Chron's disease. Med Lett Drugs Ther 1999; 41: 19-20.

7. Rovere Quercini P, Vecellio M, Sabbadini MG, Ciboddo G. Miliary tuberculosis after biological therapy for rheumatoid arthritis. Rheumatology 2002; 41: 231.

8. Vonkeman HE, van der Valk PD, Mulder L, van der Valk PD, Mulder L, van de Laar MA. Fatal miliary tuberculosis during treatment with infliximab. Ned Tijdschr Geneeskd 2002; 146: 1196-1199.

9. Mayordomo L, Marengo JL, Gomez-Mateos J, Rejon E. Pulmonary miliary tuberculosis in a patient with anti-TNF alpha treatment. Scand J Rheumatol 2002; 31: 44-45.

10. Alsoub H, Alousi FS. Miliary tuberculosis in Quatar: a review of 32 adult cases. Ann Saudi Med 2001; 21: 16-20.

11. Veiga Gonzalez M, Riestra Martinez M, Fresno Forcelledo M, Gonzalez Gonzalez M, Ablanedo Ablanedo P, Herrero Zapatero A. Miliary tuberculosis: autopsy study of 29 cases. An Med Interna 1995; 12: 17-20.

12. Mert A, Bilir M, Tabak F, Ozaras F, et al. Miliary tuberculosis: clinical manifestations, diagnosis and outcome in 38 adults. Respirology 2001; 6: 217-224.

13. Ibanez Martinez J, Bautista Ojeda MD, Saez Torrez C, Jorda I Heras M, Ayerza Lerchundi MA, Pachion Diaz J. Multicenter study of tuberculosis in clinical autopsies in Andalucia, in the 1973-1988 period. Rev Clin Esp 1991; 188: 273-277.

14. Grinsztejn B, Fandinho FC, Veloso VG, et al. Mycobacteremia in patients with the acquired immunodeficiency syndrome. Arch Intern Med 1997; 157: 23592363.

15. Lado-Lado FL, Barrio Gomez E, Carballo Arceo E, Cabarcos Ortiz de Barron A. Pulmonary tuberculosis with normal chest radiographs in HIV-immunodeficient patients. AIDS 1999; 13: 1146-1147.

16. Lado-Lado FL, Barrio Gomez E, Carballo Arceo E, Cabarcos Ortiz de Barron A. Clinical presentation of tuberculosis and the degree of immunodeficiency in patients with HIV infection. Scand J Infect Dis 1999; 31: 387-391.

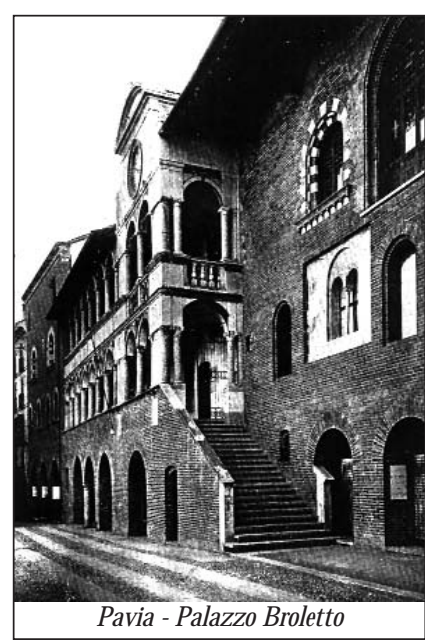

